\title{
Antidepressive effects of targeting ELK-1 signal transduction
}

\author{
Kallia Apazoglou ${ }^{1,10}$, Séverine Farley ${ }^{1,10}$, Victor Gorgievski ${ }^{1,10}$, Raoul Belzeaux ${ }^{2,3,4}$, Juan Pablo Lopez ${ }^{2}$, \\ Julien Grenier5, El Chérif Ibrahim 4,6, Marie-Anne El Khoury', Yiu C. Tse², Raphaele Mongredien', \\ Alexandre Barbé1, Carlos E. A. de Macedo', Wojciech Jaworski', Ariane Bochereau', Alejandro Orrico', \\ Elsa Isingrini' ${ }^{2}$ Chloé Guinaudie ${ }^{2}$, Lenka Mikasova7, Franck Louis' ${ }^{1}$, Sophie Gautron', Laurent Groc ${ }^{7}$, \\ Charbel Massaad ${ }^{5}$, Ferah Yildirim ${ }^{8}$, Vincent Vialou', Sylvie Dumas ${ }^{9}$, Fabio Marti ${ }^{1}$, Naguib Mechawar², \\ Elise Morice', Tak P. Wong $\mathbb{1}^{2}$, Jocelyne Caboche1, Gustavo Turecki², Bruno Giros ${ }^{1,2,4 \star}$ and Eleni T. Tzavara ${ }^{1,4 \star}$
}

\begin{abstract}
Depression, a devastating psychiatric disorder, is a leading cause of disability worldwide. Current antidepressants address specific symptoms of the disease, but there is vast room for improvement'. In this respect, new compounds that act beyond classical antidepressants to target signal transduction pathways governing synaptic plasticity and cellular resilience are highly warranted ${ }^{2-4}$. The extracellular signal-regulated kinase (ERK) pathway is implicated in mood regulation ${ }^{5-7}$, but its pleiotropic functions and lack of target specificity prohibit optimal drug development. Here, we identified the transcription factor ELK-1, an ERK downstream partner ${ }^{8}$, as a specific signaling module in the pathophysiology and treatment of depression that can be targeted independently of ERK. ELK1 mRNA was upregulated in postmortem hippocampal tissues from depressed suicides; in blood samples from depressed individuals, failure to reduce ELK1 expression was associated with resistance to treatment. In mice, hippocampal ELK-1 overexpression per se produced depressive behaviors; conversely, the selective inhibition of ELK-1 activation prevented depression-like molecular, plasticity and behavioral states induced by stress. Our work stresses the importance of target selectivity for a successful approach for signal-transduction-based antidepressants, singles out ELK-1 as a depression-relevant transducer downstream of ERK and brings proof-of-concept evidence for the druggability of ELK-1.

ERK signaling in the hippocampus has been extensively implicated in the regulation of affective responses ${ }^{9,10}$. However, because ERK controls a multitude of downstream substrates mediating diverse and even antagonistic biological functions, direct manipulation of this protein is a strategy for targeted drug development that is unlikely to be successful. On the other hand, the identification and targeted intervention of specific signaling modules that mediate stress and affective responses downstream of ERK may offer signaling-based therapeutic approaches that go beyond classical neurotransmitter-based antidepressants.
\end{abstract}

The transcription factor ELK-1 is one such candidate. In the hippocampus, ELK-1 is activated upon phosphorylation by ERK, which is triggered by two major systems that determine the impact of stressful events and their long-lasting behavioral outcomes: the glutamate receptor $N$-methyl-D-aspartate (NMDA-R) and the glucocorticoid receptor (GC-R) systems ${ }^{11,12}$. ELK-1 integrates the signals of these pathways ${ }^{13,14}$ and thus may constitute a key factor in the transition from the response to stress to the pathogenesis of depression. Accordingly, recent findings in humans show that ELK-1-regulated genes are overexpressed in individuals with major depression $^{15,16}$. However, the regulation of ELK-1 in depression and its relevance as a potential target for antidepressants has not yet been explored.

We assessed ELK1 expression in human postmortem tissues of the dentate gyrus (DG) of the left hippocampus from 28 depressed suicides and 22 matched psychiatrically healthy controls (Supplementary Table 1). This region was chosen because in postmortem and neuroimaging studies, it is consistently implicated in major depressive disorder and suicide ${ }^{17,18}$. ELK1 mRNA was significantly elevated in DG tissue from depressed suicides compared with controls (Fig. 1a). There was no increase of ELK1 expression in the prefrontal cortex (PFC; Supplementary Fig. 1). We next investigated Elk1 mRNA expression in two complementary mouse models of stress and depression-like behaviors, unpredictable chronic mild stress (UCMS $)^{19}$ and social defeat (SD) ${ }^{20,21}$. In addition to the hippocampus, other brain regions also implicated in stress and depression, including the PFC and the nucleus accumbens (NAcc), were investigated. We first performed Elk1 cartography with fluorescence in situ hybridization (FISH). In the hippocampus (of 12- to 36-week-old mice), basal Elk1 expression was highest in the DG and lowest in the CA2/3 area. In all hippocampal subfields, Elk1 was expressed in glutamatergic, but not $\gamma$-aminobutyric acid (GABA)ergic, neurons (Fig. 1b). A similar segregation of Elk1 expression in Slc17a7 (Vglut1)-positive neurons was seen in the PFC, whereas Elk1 was expressed in most GABA-ergic neurons in the NAcc

\footnotetext{
'Neuroscience Paris Seine-IBPS, INSERM UMRS 1130/CNRS UMR8246, Sorbonne University, Université Pierre et Marie Curie, Paris, France. ${ }^{2}$ Department of Psychiatry, Douglas Mental Health University Institute, McGill University Faculty of Medicine, Montreal, Quebec, Canada. ${ }^{3}$ Assistance Publique Hôpitaux de Marseille, Sainte Marguerite Hospital, Pôle de Psychiatrie Universitaire Solaris, Marseille, France. ${ }^{4}$ FondaMental Foundation, Créteil, France. ${ }^{5}$ INSERM UMR-S 1124 ERL 3649, Université Paris Descartes, Paris, France. ${ }^{6}$ Aix Marseille Université, CNRS, INT, Institut des Neurosciences de la Timone, UMR 7289, Marseille, France. ${ }^{7}$ Interdisciplinary Institute for Neuroscience, CNRS UMR 5297, Université de Bordeaux, Bordeaux, France. ${ }^{8}$ Department of Neuropsychiatry, Department of Psychiatry and Psychotherapy and NeuroCure Cluster of Excellence, Charité-Universitätsmedizin Berlin, Berlin, Germany. ${ }^{9}$ Oramacell, Paris, France. ${ }^{10}$ These authors contributed equally: Kallia Apazoglou, Séverine Farley, Victor Gorgievski. *e-mail: bruno.giros@mcgill.ca; eleni.tzavara@inserm.fr
} 
a

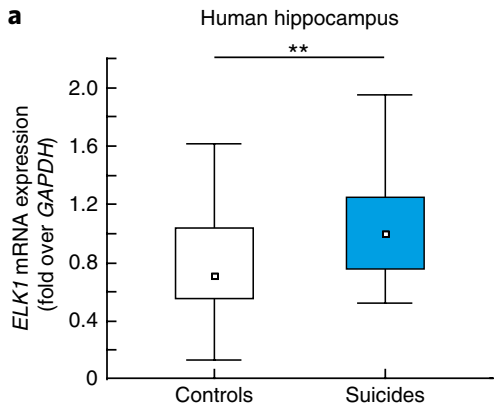

b
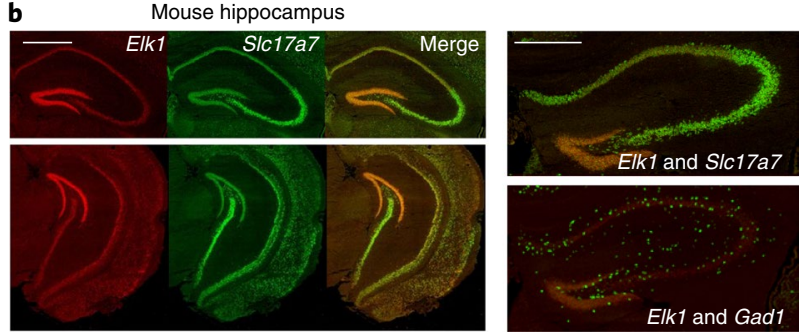

C
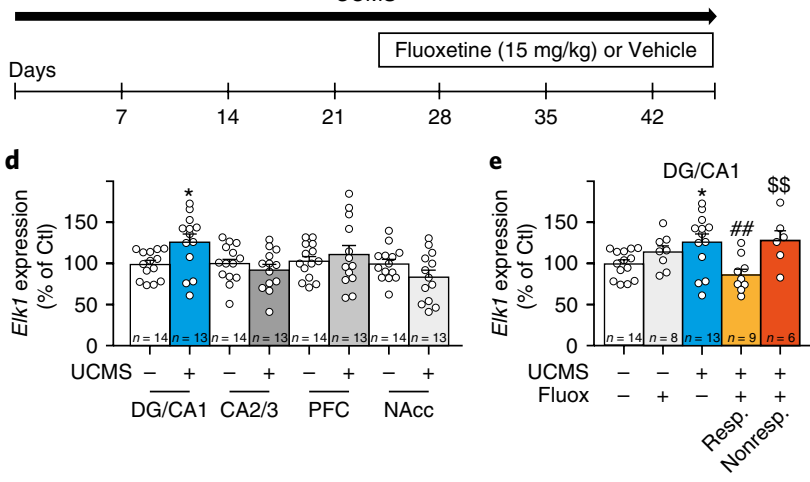

Fig. 1 | Increased hippocampal ELK1 mRNA expression in depression.

a, ELK1 mRNA expression (\% of control) in the DG of the left hippocampus from human postmortem brain tissue from depressed suicides $(n=28)$ and gender and age-matched sudden-death controls $(n=22)$ as assessed through qRT-PCR. Two-sided Student's $t$-test: ${ }^{\star \star} P=0.0087$. In box-andwhisker plots, small squares mark the median ELK1 expression, the upper and lower perimeters mark the quartiles, and the tails mark the minimum and maximum. b, Representative FISH images of S/c17a7 or Gad and Elk1 mRNA expression in mouse hippocampus. These experiments were independently run twice in control, nonstressed mice. Each experiment used $n=3$ mice per group, and results were similar between experiments. Scale bars: left, $1 \mathrm{~mm}$; right, $0.5 \mathrm{~mm}$. c, Schematic of the unpredictable chronic mild stress (UCMS) protocol. d, Quantitative comparisons of Elk1 mRNA expression in specific brain regions in nonstressed control ( $\mathrm{CtI}$ ) mice or UCMS-subjected mice as assessed by qPCR. Repeated-measures one-way ANOVA (UCMS or CtI (betweenfactor); brain region (within-factor)): interaction $\mathrm{F}(3,75)=3.46$; $P=0.02$. Duncan's post hoc: ${ }^{\star} P=0.04$ versus $C t$. e, DG/CA1 Elk1 mRNA expression in fluoxetine (Fluox)- or saline-treated nonstressed and UCMS-subjected mice showing the effect of antidepressant treatment. UCMS-subjected mice treated with fluoxetine are separated into responders (Resp.) and nonresponders (Nonresp.). One-way ANOVA: $F(4,45)=4.48 ; P=0.0039$. Duncan's post hoc: ${ }^{\star} P=0.04$ versus nonstressed, saline-treated controls; $\#=0.0039$ versus UCMS-subjected, saline-treated mice; ${ }^{\$} P<0.0029$ versus responders. Histograms show mean \pm s.e.m. of Elk1 mRNA levels in stressed mice expressed as percentage of change over controls, and individual data points are depicted as white circles. $n$ values are the number of mice in each group.
(Supplementary Fig. 2). These patterns did not change with chronic stress. Subsequent quantitative comparisons through qPCR were run on four regions of interest: DG/CA1 and CA2/3 hippocampal areas, PFC and NAcc (Supplementary Fig. 3). Quantitatively, UCMS increased Elk1 in DG/CA1, but not CA2/3, PFC or NAcc (Fig. $1 c, d)$. The same region-specific Elk1 increase was seen in the $\mathrm{SD}$ paradigm, in which phenotype severity correlated with Elk1 levels in DG/CA1 (Supplementary Fig. 4). In UCMS-subjected mice, chronic administration of the antidepressant fluoxetine distinguished between responder mice (those showing improved behavioral score across two tests, the sucrose preference and the tail suspension test (TST)) and nonresponders (Supplementary Fig. 4). Reversal of depression-like behaviors in responders was associated with normalized Elk1 levels in DG/CA1 (Fig. 1e), suggesting that reduction in $E l k 1$ expression and/or activity may be an integral part of efficient antidepressant action.

Subsequently, we quantified ELK1 mRNA expression in human blood samples from healthy controls and from depressed patients before and after antidepressant treatment ( 8 weeks with the selective serotonin reuptake inhibitor (SSRI) citalopram). Patients were classified as either nonresponders or remitters on the basis of changes in their clinical scores at the end of treatment; remission was defined as a post-treatment score of 10 or less on the 21-item Hamilton Depression Rating Scale (HDRS-21) ${ }^{22}$. Although there was no between-groups difference for basal blood ELK1 mRNA expression, remitters showed greater ELK1 change than nonresponders in response to treatment (Fig. 2a). Subsequent correlation analysis showed that greater $E L K 1$ reduction was associated with greater improvement of symptoms (Fig. 2b). Importantly, this correlation was replicated in a second independent cohort of 26 depressed individuals monitored for response to treatment in a naturalistic study (Fig. 2c). In the mouse UCMS paradigm, reversal of depressive-like behaviors with fluoxetine (Supplementary Fig. 5) was paralleled by a reduced level of blood Elk1 mRNA (Fig. 2d). Many factors (for example, inflammatory ${ }^{23}$ and metabolic ${ }^{24}$ ) may confound basal ELK-1 expression in blood; therefore, its relevance as a predictive biomarker for a depressive state seems limited. On the other hand, antidepressant-induced variation of ELK-1 appears to determine a mediator effect of antidepressant response with good translational plausibility, even though we do not know at this stage how to interpret the relationship between findings in blood and changes in brain biology. However, as an important component of neuroplasticity ${ }^{13,14}$, inflammatory ${ }^{23}$ and metabolic ${ }^{24}$ pathways, ELK-1 dysregulation holds broad explanatory value for a vast range of depression-related pathophysiological processes and may offer new avenues for novel therapeutic opportunities.

Stress-induced hippocampal ERK activation was shown previously in animal models ${ }^{14,25}$. Accordingly, we show that exposure to an acute stress (tail-suspension test, TST) increased the levels of phosphorylated ERK (p-ERK) and of its effectors p-ELK-1 and $\mathrm{p}$-mitogen- and stress-activated protein kinase (MSK) and their transcriptional targets (Supplementary Fig. 6). TST-induced immobility and TST-induced increase of hippocampal p-ELK-1 were inhibited by NMDA-R and GC-R inactivation (Supplementary Fig. 7), suggesting a pathophysiological functional link between p-ELK-1 upregulation in the hippocampus and stress-induced behavioral alterations.

We therefore hypothesized that a targeted intervention via selective inhibition of ELK-1 phosphorylation (at Ser383) downstream of ERK could reverse the deleterious effects of stress and potentially alleviate depression. To inhibit the p-ELK-1 signaling module in a specific manner, we used a brain-penetrant transactivator of transcription (TAT) peptide ${ }^{26}$ (TAT-DEF-ELK-1 peptide; TDE) that specifically impedes ERK and ELK-1 molecular interaction and subsequent ELK-1 activation by interfering with the DEF-docking domain $^{27,28}$ (Supplementary Fig. 8) and that is active in vivo when administered systemically ${ }^{29}$. 

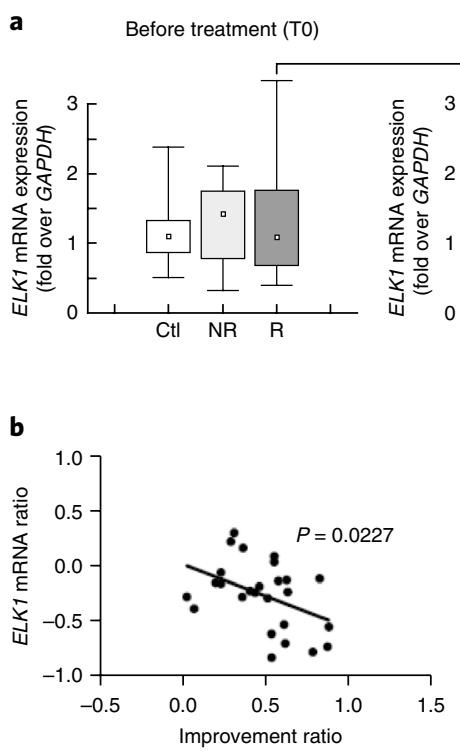

After treatment (T8)

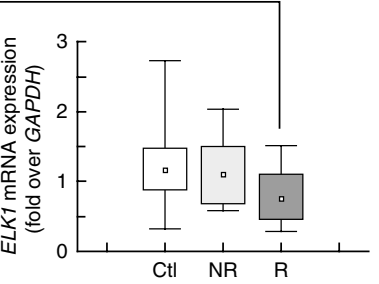

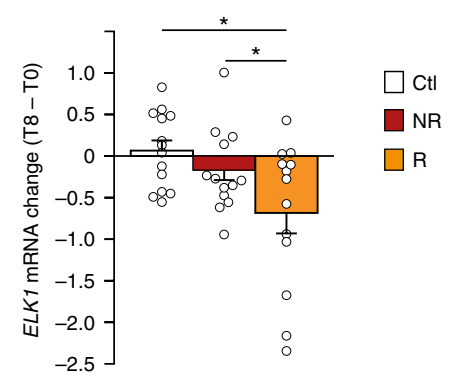

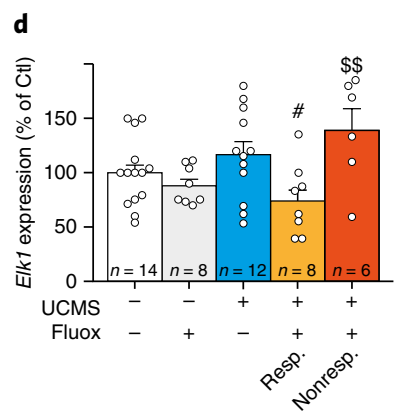

Fig. 2 | Reduction of blood ELK1 mRNA levels reflects antidepressant efficacy. a, Left, ELK1 mRNA levels (fold over GAPDH) in peripheral blood samples from the discovery cohort comprising healthy controls $(n=14)$ and depressed patients $(n=28)$ before (T0) and after (T8) antidepressant treatment ( 8 weeks citalopram) as assessed through qPCR. At T8, patients were separated into nonresponders (NR; $n=15)$ and remitters with an HDRS-21 post-treatment score $\leq 10(R ; n=13)$. The general linear model (GLM) was used for repeated measures (pre- and post-treatment) and group (Ctl, NR, R) with covariables for age, gender and depression severity at inclusion: interaction $\mathrm{F}(2,36)=4.838, P=0.014$. Paired two-sided $t$-test: ${ }^{P} P=0.017$. Right, values of $E L K 1$ change $(E L K 1-T 8-$ ELK1-TO) for each group. One-way ANOVA: $F(2,39)=5.06 ; P=0.011$. Duncan's post hoc: ${ }^{\star} P=0.004, \mathrm{R}$ versus $\mathrm{Ctl} ;{ }^{\star} P=0.036, \mathrm{R}$ versus NR. In box-and-whisker plots, small squares mark the median ELK1 expression, the upper and lower perimeters mark the quartiles, and the tails mark the minimum and maximum. b, Pearson correlation analysis between improvement ratio ((HDRS-21-T0 - HDRS-21-T8) / HDRS-21-T0) and ELK1 expression variation ratio ((ELK1-T8 - ELK1-T0) / $E L K 1-T 0)$ in the 28 patients with MDD in the discovery cohort; a greater decrease in $E L K 1$ expression correlates with better improvement $(R=-0.429, P=0.0227)$. c, Pearson correlation analysis between improvement ratio and ELK1 expression variation ratio in the replication cohort comprising 26 patients with MDD; analysis revealed a negative correlation between the ratios $(R=-0.399, P=0.0434)$. d, Blood Elk1 mRNA (\% of control) in nonstressed and UCMS-subjected mice treated with saline or fluoxetine as assessed by qPCR. One-way ANOVA: $F(4,43)=3.64 ; P=0.012$. Duncan's post hoc: $P=0.032$ versus UCMS-subjected, saline-treated mice; ${ }^{\$} P=0.001$ versus responders; $n=$ number of mice per group. Histograms show mean \pm s.e.m., and individual data points are depicted as white circles.

Fig. 3 | Selective inhibition of p-ELK-1 reverses stress-induced behavioral, molecular and plasticity changes. a, Top left, time spent immobile in the tail-suspension test (TST) following antidepressant or vehicle (Veh) treatment. TDE (i.p., 90 min before TST onset) decreased immobility similar to the reference antidepressants fluoxetine and desipramine (DMI) (mice received $15 \mathrm{mg} / \mathrm{kg}$ fluoxetine or DMI i.p. 30 min before TST onset). One-way ANOVA: $F(6,73)=16.55, P<0.00001$. Duncan's post hoc: ${ }^{\star \star} P<0.001$ versus Veh (specifically, ${ }^{\star \star} P=0.0001(0.5 \mathrm{mg} / \mathrm{kg} \mathrm{TDE}),{ }^{\star \star} P=0.00012(1 \mathrm{mg} / \mathrm{kg}$ TDE), ${ }^{\star \star} P=0.00005$ (2 mg/kg TDE), ${ }^{\star \star} P=0.000025$ (Fluox), ${ }^{\star \star} P=0.000031$ (DMI)). Top right, schematic of novelty-induced hypophagia (NIH) and results following treatment with TDE, which reduced latency to consume a palatable chocolate milk solution in a new environment. Two-sided Mann-Whitney test: ${ }^{*} P=0.035$. Middle, schematic of social defeat (SD) and behavioral scores in the social interaction (SI) and elevated plus maze (EPM) tests for control mice not subjected to SD (CtI) and SD-subjected mice, showing that TDE reversed SD-induced changes in both tests. Two-way ANOVA (between factors: $\mathrm{SD}, \mathrm{TDE})$ : interaction $\mathrm{F}(1,28)=7.28 ; P=0.011$ for $\mathrm{SI} F(1,28)=4.49, P=0.043$ for $\mathrm{EPM} ; q=0.043$ for both panels. Duncan's post hoc: ${ }^{\star \star} P<0.001$ versus CtI (specifically, ${ }^{\star \star} P=0.0046(\mathrm{SI}),{ }^{\star \star} P=0.0058\left(\right.$ EPM)); ${ }^{\#} P<0.05$ versus $\mathrm{SD}+$ Veh (specifically, ${ }^{\#} P=0.028(\mathrm{SI}),{ }^{\#} P=0.041$ (EPM)). Detailed results from the SI test are given in Supplementary Fig. 13. Bottom, schematic of the unpredictable chronic mild stress (UCMS) test and results showing sucrose preference, time spent immobile in the TST and body weight gain in nonstressed control mice (CtI) and UCMS-subjected mice at the end of the 21- $d$ UCMS protocol. TDE prevented UCMS-induced changes. Two-way ANOVA (between factors: stress, treatment): $F(1,38)=8.9, P=0.0049, q=0.0049$ for sucrose; $F(1,32)=7.76, P=0.0088, q=0.024$ for TST; $F(1,42)=4.09, P=0.05, q=0.073$ for body weight. Duncan's post hoc: ${ }^{*} P<0.001$ versus $C t \mathrm{l}$ (specifically,

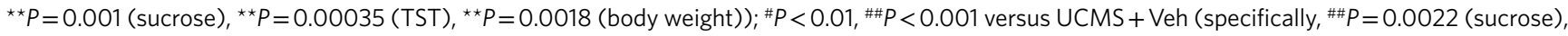
${ }^{\#} P=0.025$ (TST), \#P=0.0097 (body weight)). TDE effects using a UCMS protocol of antidepressant reversal are provided in Supplementary Fig. 14. b, Left, time course of LTP following tetanus. The change in the population field excitatory postsynaptic potential (fEPSP) slope was measured, showing synaptic plasticity in the DG in vehicle- or TDE-treated unstressed control and UCMS-subjected mice. Responses were recorded for 30 min before and $75 \mathrm{~min}$ after tetanus (which occurred at $30 \mathrm{~min}$ on the $\mathrm{x}$ axis). Each data point is an average \pm s.e.m. of 10 consecutive responses recorded over 5 min. Insert, Representative field potential before (gray) and after (black) tetanus in nonstressed control mice. Middle, Summary histograms for LTP magnitude averaged from the last $10 \mathrm{~min}$ of the $30 \mathrm{~min}$ recorded before tetanus (baseline; bl) and the last $10 \mathrm{~min}$ of recordings (LTP). Repeated-measures two-way ANOVA (time $\times$ stress $\times$ treatment): interaction $F(19,285)=2.47, P=0.00076$ for time course; $F(1,16)=8.2, P=0.011$ for histograms. LSD post hoc tests for histograms: ${ }^{\star} P=0.016,{ }^{\star \star} P<0.005,{ }^{\star \star \star} P=0.00001$ versus respective bl; ${ }^{\#} P=0.046$ versus nonstressed control (Control + Veh); ${ }^{\$ \$ \$} P=0.00015$ versus UCMS + Veh. Right, Bdnf expression (in situ hybridization) in representative images of the hippocampus in vehicle-treated nonstressed controls and vehicle- or TDE-treated UCMS-subjected mice at the end of the 21-d UCMS protocol. Quantification of Bdnf expression is also shown in a histogram. One-way ANOVA: $F(2,12)=5.97, P=0.016$ for $C A 1 ; F(2,12)=8.72, P=0.0046$ for $C A 2 / 3 ; F(2,12)=5.3, P=0.022$ for $D G$. Duncan's post hoc: for $C A 1$,

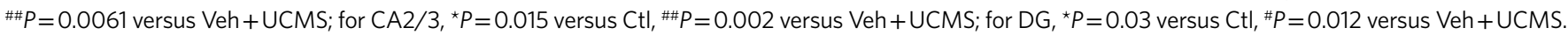
Histograms show mean \pm s.e.m., and individual data points are depicted as white circles. $n$ indicates the number of mice per group. In $\mathbf{b}, n=5$ mice per group. 
${ }_{\text {TST }}$
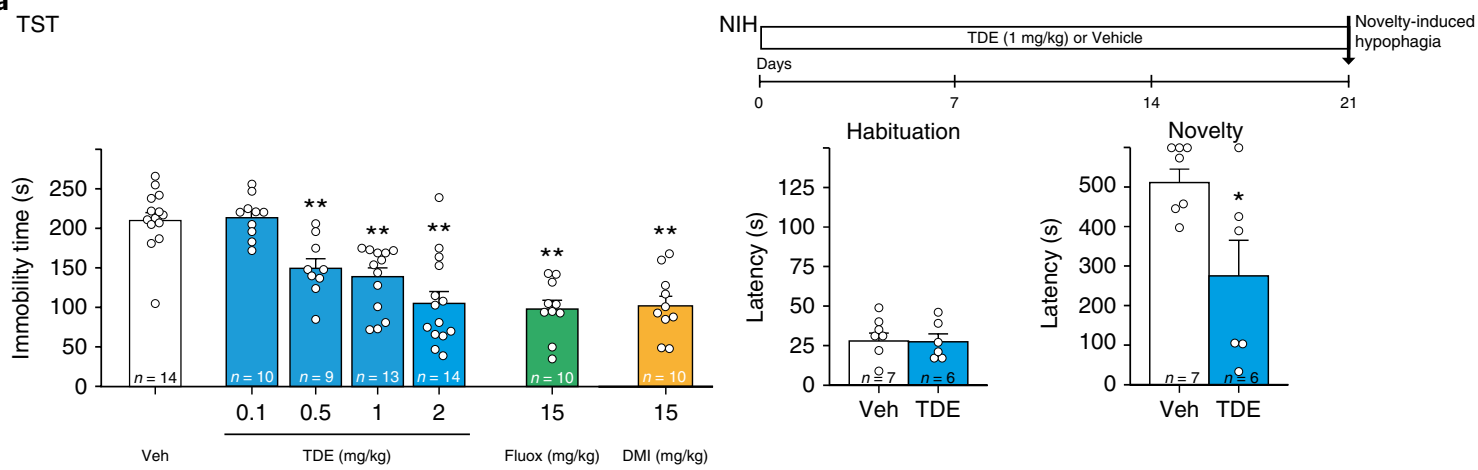

SD
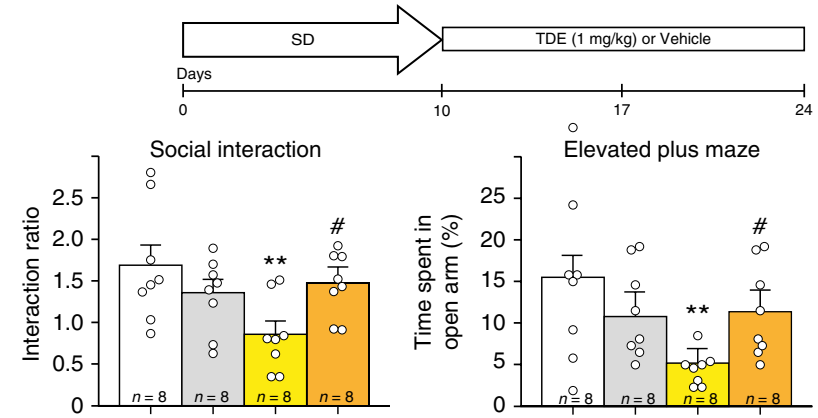

$\square \mathrm{Ctl}$

$\square \mathrm{Ctl}+\mathrm{TDE}$

$\square \mathrm{SD}+\mathrm{Veh}$

$\square \mathrm{SD}+\mathrm{TDE}$
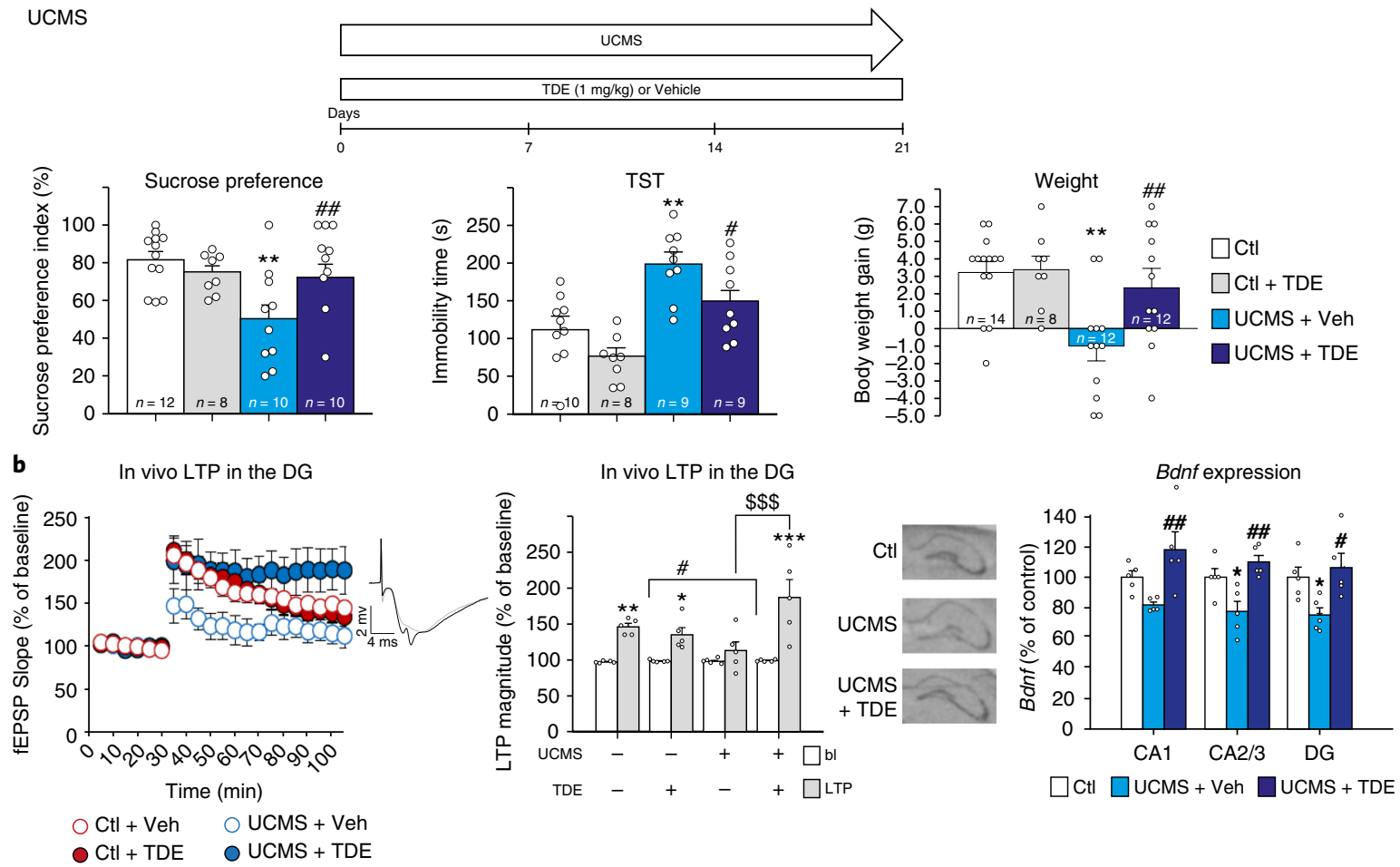

We assessed the effects of TDE-mediated inhibition of p-ELK-1 on stress-induced behavioral, plasticity and molecular changes in a mouse model (Fig. 3 and Supplementary Figs. 9-17). In all experiments, 3- to 6-month old adult male mice were used. For acute systemic administration, TDE was injected intraperitoneally (i.p.) 90 min before behavioral testing. For chronic administration, TDE was injected i.p. at a dose of $1 \mathrm{mg}$ per $\mathrm{kg}$ body weight $(\mathrm{mg} / \mathrm{kg})$ daily for 14-21 d in either a prophylactic or a reversal mode starting at the beginning of the stress-induced depression protocol or starting after stress-induced effects were seen, respectively. Acute systemic TDE administration decreased immobility in the forced-swimming test and the TST, similar to clinically active antidepressants ${ }^{30,31}$ (Fig. 3a), and prevented TST-stress-triggered cognitive deficits in a hippocampus-dependent learning paradigm ${ }^{32}$ (Supplementary Fig. 9). At the molecular level, these effects were underpinned by a selective inhibition of TST-induced phosphorylation and activation of ELK-1 (but not of ERK or MSK1) along with a concomitant reduction in the expression of Zif268, a direct downstream target of ELK-1 (Supplementary Figs. 10 and 11). We further validated ELK-1 as an antidepressant target in several paradigms, including novelty-induced hypophagia, SD and UCMS (Fig. 3a and Supplementary Figs. 12-14). In all models, TDE administration reversed stress-induced behavioral alterations. TDE had no effect on basal levels of locomotion, memory or anxiety (Supplementary Fig. 15), suggesting a targeted action on stress-triggered processes and an antidepressant-like profile that could preferentially involve 
the hippocampus, as intrahippocampal but not intra-accumbal TDE infusion also reduced TST immobility with no effect on locomotion (Supplementary Fig. 16).

We then sought to investigate how the effect of TDE administration on hippocampal function might be linked to the antidepressant action of this peptide. Major depressive disorder (MDD) is currently conceptualized as a disorder of impaired synaptic plasticity and neurotrophic signaling, in part through the GC-R and/or NMDA-R pathways ${ }^{33,34}$. As shown above, ELK-1 activation is a key mediator of GC-R and NMDA-R signals. Therefore, we investigated the ability of TDE to counteract stress-induced alterations of long-term potentiation (LTP), a depression-relevant index of dysregulated neuroplasticity ${ }^{35}$, and of brain-derived neurotrophic factor (BDNF) expression. Acute in vivo electrophysiology at the end of the UCMS protocol revealed stressimpaired LTP at the medial perforant path to dentate granule cells, which was in accordance with previous findings in hippocampal slices ${ }^{35}$. TDE restored LTP in stressed mice with no effect in controls (Fig. 3b). Similarly, TDE prevented the stress-induced reduction of Bdnf mRNA (Fig. $3 \mathrm{~b}$ and Supplementary Fig. 17). In treatment-naive, nonstressed mice, chronic administration of TDE increased expression of Bdnf mRNA (Supplementary Fig. 18), as was previously reported with classical and putative antidepressants ${ }^{34}$.

These results further implicate increased hippocampal ELK-1 signaling in the emergence and manifestation of depression-like states. To directly demonstrate such a causal link, we used a viral vector (adeno-associated virus (AAV)) to locally overexpress ELK-1 in the brains of mice. AAV-ELK-1 overexpression in the DG/CA1 hippocampal area (but not in NAcc) was sufficient to produce a depressive-like phenotype ${ }^{36}$ in treatment-naive, nonstressed mice (Fig. 4). As expected, TDE rescued depressive-like behaviors in these mice (Supplementary Fig. 19).

Taken together, our findings suggest a stress-depression framework in which increases in ELK-1 expression and/or activity

Fig. 4 | ELK-1 overexpression in the hippocampus induces depressivelike behaviors. a, Mice were injected in the hippocampus (Hipp) or NAcc with AAV-CtI or AAV-ELK-1 constructs expressing mCherry as a reporter. Representative fluorescence immunocytochemistry images show mCherry expression in mice injected with AAV-ELK1 in the hippocampus (left) or NAcc (right). 3,3'-diaminobenzidine (DAB) immunocytochemistry staining of ELK-1 in hippocampus (left) or NAcc (right) from mice injected with AAV-Ctl or AAV-ELK-1. Representative images of $n=4$ mice per group (four images per mouse). In all AAV-ELK-1-Hipp and AAV-ELK-1-NAcc mice, AAV-ELK-1 infusion resulted in the appearance of strongly ELK-1-positive cells. Scale bars, $0.2 \mathrm{~mm}$. b, Top, results for sucrose preference, time spent immobile in the TST, time spent in light in the light-dark test, and the number of ambulations in mice injected with AAV-CtI or AAV-ELK-1 in the hippocampus or NAcc. Two-way ANOVA (between factors: AAV-CtI or AAV-ELK-1, region): $F(1,31)=4.18, P=0.049$ for sucrose; $F(1,32)=15.67$, $P=0.00039$ for TST. Duncan's post hoc: ${ }^{*} P=0.014,{ }^{\star \star *} P=0.00013$ for AAV-ELK-1-Hipp versus AAV-Ctl-Hipp. Bottom, Z-normalization and computation of a global index of depressive-like behavior through transforming sucrose preference, TST immobility and time in light data into Z-scores and averaging these scores. AAV overexpression in the hippocampus (but not in the NAcc) alters behavior across dimensions (repeated-measures two-way ANOVA (dimension $\times$ AAV-CtI or AAVELK-1 $\times$ region): $F(2,62)=3.43, P=0.038$; Duncan's post hoc: ${ }^{\star} P=0.043$, ${ }^{\star \star \star} P=0.000026$ for AAV-ELK-1-Hipp versus AAV-CtI-Hipp) and induces a depressive-like profile of behavior (two-way ANOVA (between factors: AAV-Ctl or AAV-ELK-1, region): $F(1,31)=37.67, P=0.00001$. Duncan's post hoc: ${ }^{\star \star \star} P=0.000062$ for AAV-Elk-1-Hipp versus AAV-CTL-Hipp). Histograms show mean \pm s.e.m., and individual data points are depicted as white circles. $n$ indicates the number of mice per group. transduce stress-activated states, whereas ELK-1 normalization prevents stress effects on neuroplasticity, producing an antidepressant-like action.

At first sight, our results may appear paradoxical under the current frame in which reduced activity of the ERK pathway via either downregulated $\mathrm{ERK}^{37}$ or upregulated mitogen-activated protein kinase phosphatase 1 (MKP-1, also known as DUSP-1; the phosphatase that deactivates $\mathrm{ERK}^{10}$ ) is associated with depression. This apparent contradiction can be addressed at different levels. First, the prodepressant effects of increased ELK-1 signaling shown here are in direct contrast to the purported antidepressant role of the MSK1-CREB cascade also operating downstream of ERK ${ }^{31,38,39}$. A similar dichotomy between the MSK1-CREB cascade and ELK-1 modules, which act antagonistically downstream of ERK, has been shown in other psychiatric conditions, such as addiction ${ }^{26,29,40}$, and highlights the importance of target selectivity for optimal signaling-based pharmacological interventions. Accordingly, and in line b Sucrose preference
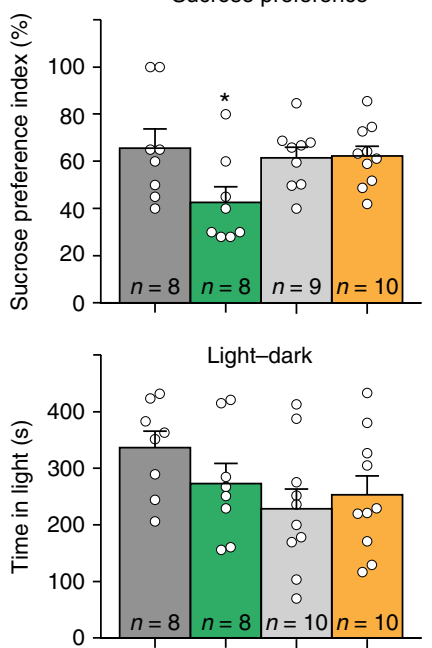

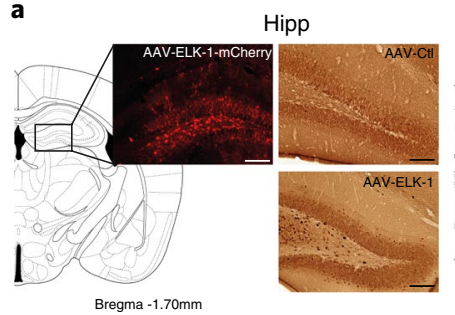

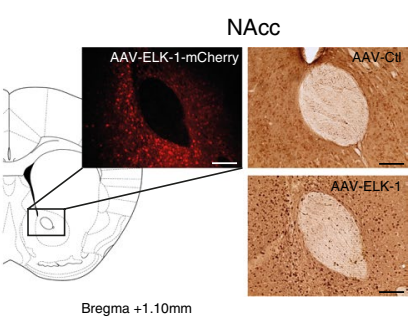

TST
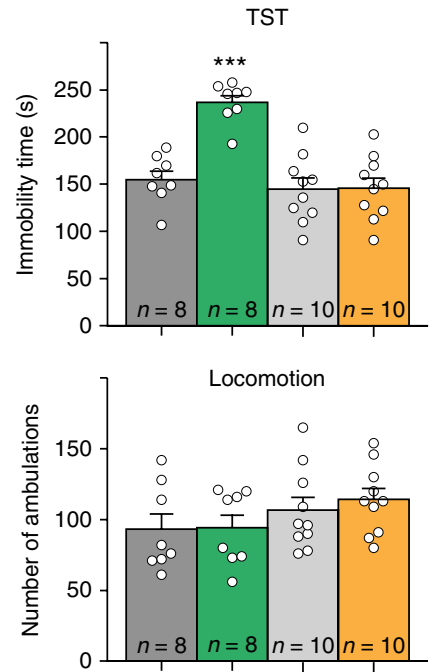
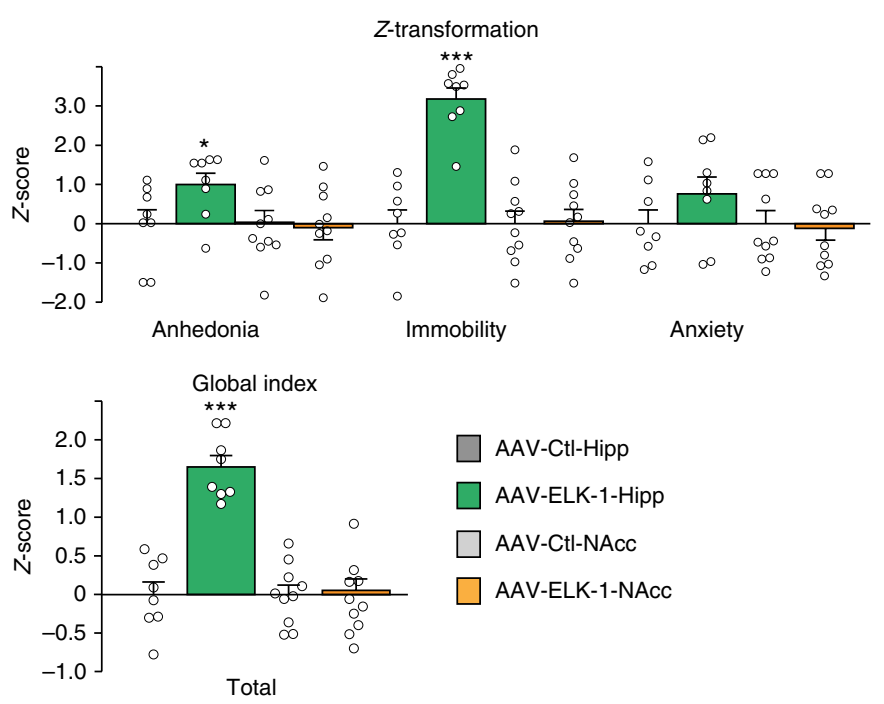
with previous findings ${ }^{5}$, SL327, an inhibitor of MEK (the kinase that phosphorylates and activates ERK), which nonselectively inhibits all downstream actions of $\mathrm{ERK}^{26}$, failed to reduce immobility in the TST (Supplementary Fig. 20). Second, as we show in the SD model (Supplementary Fig. 21), ELK-1 inhibition reverses the depressionrelated induction of the phosphatase DUSP1, further orienting the activated network state toward an antidepressant response ${ }^{10}$ that is mechanistically coupled to restored BDNF signaling ${ }^{34}$.

Overall, we demonstrate that the functional consequences of selective ELK-1 inhibition are markedly different from those of global ERK inhibition, offering a revised and promising signaltransduction-based concept for understanding and managing depression. Reaching beyond ERK, we identify ELK-1 as a key prodepressant mediator that can be dissociated from other ERK effectors and therefore is targetable and druggable. Compared to classical monoaminergic antidepressants, blockade of ELK-1 activation with TDE has the advantage of acting beyond the synaptic and/or neurotransmitter level to directly change the balance and orientation of intracellular signaling. Future studies are warranted to explore whether this new mechanism of action could also translate into enhanced or facilitated response to antidepressants used clinically.

\section{Methods}

Methods, including statements of data availability and any associated accession codes and references, are available at https://doi. org/10.1038/s41591-018-0011-0.

Received: 26 September 2016; Accepted: 12 February 2018; Published online: 07 May 2018

\section{References}

1. Perlis, R. H. Abandoning personalization to get to precision in the pharmacotherapy of depression. World Psychiatry 15, 228-235 (2016).

2. Ionescu, D. F. \& Papakostas, G. I. Experimental medication treatment approaches for depression. Transl. Psychiatry 7, e1068 (2017).

3. Yuan, L. L., Wauson, E. \& Duric, V. Kinase-mediated signaling cascades in mood disorders and antidepressant treatment. J. Neurogenet. 30, 178-184 (2016).

4. Jeon, S. W. \& Kim, Y. K. Molecular neurobiology and promising new treatment in depression. Int. J. Mol. Sci. 17, 381 (2016).

5. Duman, C. H., Schlesinger, L., Kodama, M., Russell, D. S. \& Duman, R. S. A role for MAP kinase signaling in behavioral models of depression and antidepressant treatment. Biol. Psychiatry 61, 661-670 (2007).

6. Einat, $\mathrm{H}$. et al. The role of the extracellular signal-regulated kinase signaling pathway in mood modulation. J. Neurosci. 23, 7311-7316 (2003).

7. Labonté, B. et al. Sex-specific transcriptional signatures in human depression. Nat. Med. 23, 1102-1111 (2017).

8. Janknecht, R., Ernst, W. H., Pingoud, V. \& Nordheim, A. Activation of ternary complex factor Elk-1 by MAP kinases. EMBO J. 12, 5097-5104 (1993).

9. Gould, T. D. \& Manji, H. K. Signaling networks in the pathophysiology and treatment of mood disorders. J. Psychosom. Res. 53, 687-697 (2002).

10. Duric, V. et al. A negative regulator of MAP kinase causes depressive behavior. Nat. Med. 16, 1328-1332 (2010).

11. Popoli, M., Yan, Z., McEwen, B. S. \& Sanacora, G. The stressed synapse: the impact of stress and glucocorticoids on glutamate transmission. Nat. Rev. Neurosci. 13, 22-37 (2011).

12. Tse, Y. C., Bagot, R. C., Hutter, J. A., Wong, A. S. \& Wong, T. P. Modulation of synaptic plasticity by stress hormone associates with plastic alteration of synaptic NMDA receptor in the adult hippocampus. PLoS One 6, e27215 (2011).

13. Revest, J. M. et al. The MAPK pathway and Egr-1 mediate stress-related behavioral effects of glucocorticoids. Nat. Neurosci. 8, 664-672 (2005).

14. Gutièrrez-Mecinas, M. et al. Long-lasting behavioral responses to stress involve a direct interaction of glucocorticoid receptors with ERK1/2-MSK1Elk-1 signaling. Proc. Natl. Acad. Sci. USA 108, 13806-13811 (2011).

15. Belzeaux, R. et al. Responder and nonresponder patients exhibit different peripheral transcriptional signatures during major depressive episode. Transl. Psychiatry 2, e185 (2012).

16. Menke, A. et al. Dexamethasone stimulated gene expression in peripheral blood is a sensitive marker for glucocorticoid receptor resistance in depressed patients. Neuropsychopharmacology 37, 1455-1464 (2012).

17. Vythilingam, M. et al. Childhood trauma associated with smaller hippocampal volume in women with major depression. Am. J. Psychiatry 159, 2072-2080 (2002).
18. Turecki, G., Ernst, C., Jollant, F., Labonté, B. \& Mechawar, N. The neurodevelopmental origins of suicidal behavior. Trends Neurosci. 35, $14-23$ (2012).

19. Farley, S., Apazoglou, K., Witkin, J. M., Giros, B. \& Tzavara, E. T. Antidepressant-like effects of an AMPA receptor potentiator under a chronic mild stress paradigm. Int. J. Neuropsychopharmacol. 13, 1207-1218 (2010).

20. Tsankova, N. M. et al. Sustained hippocampal chromatin regulation in a mouse model of depression and antidepressant action. Nat. Neurosci. 9, 519-525 (2006)

21. Krishnan, V. et al. Molecular adaptations underlying susceptibility and resistance to social defeat in brain reward regions. Cell 131, 391-404 (2007).

22. Lopez, J. P. et al. miR-1202 is a primate-specific and brain-enriched microRNA involved in major depression and antidepressant treatment. Nat. Med. 20, 764-768 (2014).

23. Nilsson, M. et al. Elk1 and SRF transcription factors convey basal transcription and mediate glucose response via their binding sites in the human LXRB gene promoter. Nucleic Acids Res. 35, 4858-4868 (2007).

24. Chen, E., Miller, G. E., Kobor, M. S. \& Cole, S. W. Maternal warmth buffers the effects of low early-life socioeconomic status on pro-inflammatory signaling in adulthood. Mol. Psychiatry 16, 729-737 (2011).

25. Yang, C. H., Huang, C. C. \& Hsu, K. S. A critical role for protein tyrosine phosphatase nonreceptor type 5 in determining individual susceptibility to develop stress-related cognitive and morphological changes. J. Neurosci. 32, 7550-7562 (2012).

26. Lavaur, J. et al. A TAT-DEF-Elk-1 peptide regulates the cytonuclear trafficking of Elk-1 and controls cytoskeleton dynamics. J. Neurosci. 27, 14448-14458 (2007).

27. Jacobs, D., Glossip, D., Xing, H., Muslin, A. J. \& Kornfeld, K. Multiple docking sites on substrate proteins form a modular system that mediates recognition by ERK MAP kinase. Genes Dev. 13, 163-175 (1999).

28. Sharrocks, A. D., Yang, S. H. \& Galanis, A. Docking domains and substrate-specificity determination for MAP kinases. Trends Biochem. Sci. 25, 448-453 (2000).

29. Besnard, A. et al. Alterations of molecular and behavioral responses to cocaine by selective inhibition of Elk-1 phosphorylation. J. Neurosci. 31, 14296-14307 (2011).

30. Crozatier, C. et al. Calcineurin (protein phosphatase 2B) is involved in the mechanisms of action of antidepressants. Neuroscience 144, 1470-1476 (2007).

31. Svenningsson, P. et al. Involvement of striatal and extrastriatal DARPP-32 in biochemical and behavioral effects of fluoxetine (Prozac). Proc. Natl. Acad. Sci. USA 99, 3182-3187 (2002).

32. Mouri, A. et al. Involvement of a dysfunctional dopamine-D1/N-methyl-daspartate-NR1 and $\mathrm{Ca}_{2}+$ calmodulin-dependent protein kinase II pathway in the impairment of latent learning in a model of schizophrenia induced by phencyclidine. Mol. Pharmacol. 71, 1598-1609 (2007).

33. Manji, H. K. et al. Enhancing neuronal plasticity and cellular resilience to develop novel, improved therapeutics for difficult-to-treat depression. Biol. Psychiatry 53, 707-742 (2003).

34. Duman, R. S., Aghajanian, G. K., Sanacora, G. \& Krystal, J. H. Synaptic plasticity and depression: new insights from stress and rapid-acting antidepressants. Nat. Med. 22, 238-249 (2016).

35. Nasca, C. et al. Stress dynamically regulates behavior and glutamatergic gene expression in hippocampus by opening a window of epigenetic plasticity. Proc. Natl. Acad. Sci. USA 112, 14960-14965 (2015).

36. Guilloux, J. P., Seney, M., Edgar, N. \& Sibille, E. Integrated behavioral $z$-scoring increases the sensitivity and reliability of behavioral phenotyping in mice: relevance to emotionality and sex. J. Neurosci. Methods 197, 21-31 (2011).

37. Dwivedi, Y. et al. Reduced activation and expression of ERK1/2 MAP kinase in the post-mortem brain of depressed suicide subjects. J. Neurochem. 77, 916-928 (2001).

38. Qi, X. et al. Fluoxetine increases the activity of the ERK-CREB signal system and alleviates the depressive-like behavior in rats exposed to chronic forced swim stress. Neurobiol. Dis. 31, 278-285 (2008).

39. Svenningsson, P. et al. Biochemical and behavioral evidence for antidepressant-like effects of 5-HT6 receptor stimulation. J. Neurosci. 27, 4201-4209 (2007).

40. Brami-Cherrier, K. et al. Parsing molecular and behavioral effects of cocaine in mitogen- and stress-activated protein kinase-1-deficient mice. J. Neurosci. $25,11444-11454$ (2005).

\section{Acknowledgements}

We thank P. Greengard, G. G. Nomikos and R. H. Perlis for critical reading of the manuscript. We want to acknowledge M. -J. Brisorgueil and A. Besnard for help with immunohistochemistry experiments, C. Tecker for qPCR experiments in mice, P. Vanhoutte for TDE design and the Imaging Platform at the Institut de la Vision (Paris, France) for slide scanning. This research was supported in part by ERANET NEURON (Grant WM2NA; N.M., C.M., E.T.T.), Labex-Biopsy (AIM: E.T.T.; SignAddict: J.C.), FRC (Fondation Recherche pour le Cerveau; E.T.T.), the National Hospital Clinical Research Program (Assistance Publique-Hopitaux de Marseille; 
PHRC no. 2010-19: R.B.), Conseil Régional d'Aquitaine (L.M., L.G.) and the Canadian Institute for Health Research (B.G. and G.T.). R.B. was supported by a FondaMental Servier postdoctoral fellowship, and K.A. was supported by an MRT (Ministère Recherche Technologie, Ecole Doctorale MTCI) graduate award. B.G. is the holder of the Graham Boeckh Chair, and E.T.T. is a past recipient of the Bodossakis Foundation Young Scientist Award.

\section{Author contributions}

E.T.T. and B.G. designed the study with the help of V.G. and J.C. for mouse studies and R.B. and G.T. for clinical studies; K.A., S.F., V.G. and M.-A.E.K. performed experiments and analyzed data in mouse studies; R.M., A. Barbé, C.E.A.d.M., W.J., A. Bochereau, A.O., E.I., C.G., L.M., F.L., S.G., L.G. and V.V. performed experiments in mice; R.B., N.M. and G.T. contributed clinical and postmortem samples; R.B., J.P.L. and E.C.I. generated and analyzed clinical and postmortem data; E.M. and F.M. generated in vivo electrophysiological data; Y.C.T. and T.P.W. performed electrophysiological experiments; J.G., E.C.I. and C.M. performed qPCR experiments; S.D. and S.F. performed FISH experiments; F.Y. designed and provided viral vectors; J.C. designed and provided pharmacological tools; E.T.T. also participated in analyses of behavioral and clinical data; V.G., R.B., J.G., E.C.I., N.M., F.M., B.G. and E.T.T. wrote the paper.

\section{Competing interests}

The TDE peptide used in the present article and its application in depression are protected by the published patents WO 2006/087242 and WO2010/037841, respectively, filed in Europe, the United States, Canada and Japan. This does not alter our adherence to Nature Publishing Group policies on sharing data and materials. J.C., B.G. and E.T.T. are founding shareholders of Melkin Pharmaceuticals, a biotech company, which has developed the TDE peptide as a drug candidate. The company did not have any role in study design, data collection and analysis, decision to publish or preparation of the manuscript.

\section{Additional information}

Supplementary information is available for this paper at https://doi.org/10.1038/ s41591-018-0011-0.

Reprints and permissions information is available at www.nature.com/reprints. Correspondence and requests for materials should be addressed to B.G. or E.T.T.

Publisher's note: Springer Nature remains neutral with regard to jurisdictional claims in published maps and institutional affiliations. 


\section{Methods}

Clinical studies and molecular determinations in human tissues. Ethical approval for this study was obtained from the Institutional Review Board, Douglas Mental Health University Institute, McGill University, Montreal and CPP Sud Méditerranée II, Marseille, France for the replication cohort, and all relevant ethical regulations were followed. Informed written consent was obtained from all participants in this study or from next of kin in the case of postmortem tissue.

Postmortem human brain samples. Ethical approval for this study was obtained from the research ethics board at the Douglas Mental Health University Institute, Montreal, and all relevant ethical regulations were followed. Postmortem hippocampal samples were obtained from the Douglas-Bell Canada Brain Bank in collaboration with the Quebec Coroner's Office. Psychological autopsies were performed for all subjects based on Diagnostic and Statistical Manual of Mental Disorders, fourth edition (DSM-IV) criteria, as described previously. The two subject groups consisted of depressed suicides $(n=28)$ and age-matched suddendeath controls $(n=22)$; all subjects were male. Frozen hippocampus (DG) samples from the left hemisphere were carefully dissected by experienced histopathologists using reference neuroanatomical maps ${ }^{41}$. Additional information is given in the Supplementary Note.

Clinical study and collection of human blood samples. Discovery cohort. Subjects were recruited at a community outpatient clinic at the Douglas Mental Health University Institute. Exclusion criteria were: (i) comorbidity with other major psychiatric disorders, (ii) positive tests for illicit drugs at any point during the study and (iii) general medical illnesses. Control subjects were also excluded if they had a history of antidepressant treatment. Patients with MDD included in the study were individuals with a diagnosis of MDD without psychotic features, as determined according to the DSM-IV, who were untreated at time of inclusion (T0). Patients were treated with citalopram for 8 weeks, starting at T0 with an initial dose of $10 \mathrm{mg} / \mathrm{d}$, which was titrated progressively to a maximum of $60 \mathrm{mg} / \mathrm{d}$. Depression severity was assessed at inclusion (T0) and after 8 weeks (T8) with the HDRS21. Control subjects, treated patients and their characteristics were previously described $^{22}$. Treatment compliance was assessed using high-performance liquid chromatography at the end of the trial. All treated patients showed detectable plasma citalopram levels, and we observed a correlation between citalopram dose and plasma concentration.

Overall, 14 healthy controls $(42.7 \%$ female, $38.1 \pm 9.4$ years of age (mean \pm s.d. $)$ ) and 28 patients with MDD ( $67.9 \%$ female, $45.0 \pm 10.9$ years of age (mean \pm s.d.)) were included in this analysis. In patients, the mean depression severity score was 25.1 ( \pm 5.7 s.d.) as determined by the HDRS-21. Mean improvement ratio was calculated using the following formula: ((HDRS-21 score at T0 (HDRS-21-T0)) (HDRS-21-T8)) / (HDRS-21-T0)). The mean improvement ratio was $49.3 \pm 23.0 \%$ (s.d.). The normality of distribution of ELK1 mRNA expression value was confirmed by (skewness / s.d.) <3. Repeated measures (before and after treatment) were analyzed using a paired $t$-test. Moreover, group by time interactions were evaluated using GLM for repeated measures.

The effects of the following potentially relevant confounds were assessed statistically: severity of depression at inclusion (HDRS-21-T0), sex and age. There was no significant main effect. Including these parameters as covariables in the GLM model did not change our conclusions (GLM for repeated measures ELK1 mRNA expression value pre- and post-treatment and group (Ctl, nonresponders, remitters) interaction: $\mathrm{F}(2,37)=8.299 ; P=0.001$ and $\mathrm{F}(2,34)=7.405 ; P=0.002$ when including age, gender and depression severity at inclusion as covariables).

Then, we used the Pearson correlation analysis to determine the relationship between $E L K 1$ expression variation across time ((ELK1 expression at T8 (ELK1-T8) - ELK1-T0) / ELK1-T0) and improvement ratio; two subjects with $E L K 1$ variation $>2$ s.d. beyond the mean were excluded from correlation analyses. We also analyzed the correlation between HDRS-21 score and ELK1 mRNA level in blood before (T0) and after (T8) treatment only in samples from treated patients. We found no correlation between ELK1 mRNA before treatment $(R=-0.045$, $P=0.820)$. We found a significant positive correlation between these measures 8 weeks later $(R=0.424, P=0.025)$. This correlation is in line with our findings of a specific decrease of ELK1 mRNA after treatment in responders only. Moreover, there was no correlation between ELK1 mRNA T0 level and improvement ratio $(R=0.031, P=0.874)$, and we found no predictive value of $E L K 1 \mathrm{mRNA}$ at T0 for remission (receiver operating characteristic (ROC) curve analysis: area under the curve $($ AUC $)=0.528, P=0.800)$.

Replication cohort. Eligible study participants were part of a larger naturalistic multicentric cohort of adults diagnosed with a current MDE (major depressive episode) from a study registered at ClinicalTrials.gov with ID: NCT02209142. We used 86 healthy controls ( 32 men with a mean age of $44.5 \pm 15.1$ years (s.d.); 54 women with a mean age of $44.3 \pm 13.6$ years (s.d.)) of this cohort as the calibrator sample.

Participants underwent a clinical evaluation using the Semi-Structured Clinical Interview for DSM-IV (SCID). Patients were recently admitted in a psychiatric unit or were recently referred to a psychiatrist for a MDE. MDE diagnosis, according to the DSM-IV criteria of mood disorders, was confirmed by trained psychiatrists, and MDE severity was evaluated by the 17-item Hamilton Depression Rating Scale (HDRS-17). Patients were included if they scored 19 or higher on the HDRS-17.

Exclusion criteria were a history of bipolar disorder, patients receiving lithium, anticonvulsants or antipsychotics during the study, substance-use disorder in the past 12 months, a diagnosis of schizophrenia, psychotic or schizoaffective disorder according to the DSM-IV, a severe progressive medical disease, pregnancy, vaccination within a month before the inclusion in the study and being under 18. Moreover, based on the involvement of ELK-1 in immunological processes and the potentially confounding interaction between chronic mild inflammation and antidepressant respons $\mathrm{e}^{42}$, we excluded from this analysis all patients with a serum C-reactive protein (CRP) level $>5 \mathrm{mg} / \mathrm{L}$ at inclusion and/or patients with a variation in CRP level exceeding threefold (a criterion for inflammation ${ }^{43}$ between inclusion and week 8).

Overall, we analyzed ELK1 mRNA expression at inclusion and 8 weeks later in 26 patients with MDE ( 16 women, $61.5 \%$ ) with a mean age of $41.7 \pm 17.0$ years (s.d.) and a mean depressive symptoms severity score of $23 \pm 3.2$ (s.d.). The mean percent of improvement was $42 \pm 30 \%$ (s.d.). 11 subjects were free of treatment at the inclusion, and 15 were already receiving an antidepressant treatment. Patients were treated and followed-up according to the choice of their treating psychiatrist, using a SSRI $(n=11)$, selective serotonin-norepinephrine reuptake inhibitor (SNRI; $n=9$ ) or another antidepressant (i.e., tricyclic, agomelatine or MAOI). We computed Pearson correlation analysis to determine the relationship between ELK1 expression variation across time and improvement ratio.

RNA extraction and qRT-PCR for discovery ${ }^{22}$ and replication ${ }^{15,44,45}$ cohorts were performed as previously published and are described in detail in the Supplementary Note.

Behavioral studies and biochemical determinations in mice. Mice. All mice used were male adults (aged 3-6 months). The following strains were used: C57Bl6, $\mathrm{CD} 1$ and BALB/cJico. Details are provided in the Supplementary Note. The mice were kept under standard conditions of a 12 -h light-dark cycle at $22 \pm 1{ }^{\circ} \mathrm{C}$ with food and water available ad libitum except when food and/or water deprivation was part of the experimental protocol. Behavioral assessments were performed during the second half of the light phase. During the UCMS prophylactic and reversal protocols, treatment was administered before switching to the dark phase. All protocols and animal welfare complied with French and European Ethical regulations. The experimental protocols were approved by the local Ethical Committee (Comité d'éthique en expérimentation animale Charles Darwin No. 5; protocol number 01487).

TDE and scrambled peptide. The TDE peptide (TAT-DEF-Elk-1: GRKKRRQRRRPPSPAKLSFQFPSSGSAQVHI) as well as the scrambled peptide (GRKKRRQRRRPPQSKPSGSQHPIFSLAFVAS) were synthesized by the platform services of the University Pierre and Marie Curie (UPMC; Paris, France).

Generation of adeno-associated viruses for ELK-1 overexpression. ELK-1 (wild type) cDNA along with mCherry cDNA linked to an IRES fragment were cloned into a recombinant $\mathrm{AAV} 2 / 1$ expression vector containing a cytomegalovirus (CMV) promoter. The control vector expressed mCherry under the control of the same promoter (AAV2/1-mCherry). Virus generation and purification (performed by the University of Iowa Vector Core) yielded viruses with final titers of $2.54 \times 10^{13}$ vector genomes (VG) $/ \mathrm{ml}$ and $1.66 \times 10^{13} \mathrm{VG} / \mathrm{ml}$ for the AAV2/1ELK-1-wt and AAV2/1-mCherry viruses, respectively. Protocols for the stereotaxic surgery ${ }^{46}$ that was performed according to the atlas of Paxinos and Franklin ${ }^{47}$, for histological assessment and for behavioral testing and analysis are described in the Supplementary Note.

Behavioral studies. Behavioral dimensions and cognitive functions were assessed through the TST ${ }^{30,31}$, the FST ${ }^{48}$, the elevated plus maze, and the light-dark test ${ }^{49}$ as well as tests for novelty-induced hypophagia ${ }^{50,51}$, sucrose $_{\text {preference }}{ }^{19}$, latent learning ${ }^{32}$ and novel-object recognition ${ }^{52}$ according to previously described protocols with minor modifications. Locomotor activity was assessed in an automated actimeter ${ }^{53}$. Procedures are described in detail in the Supplementary Note. Procedures for intracerebral infusions of pharmacological compounds (TDE, ERK inhibitor (ERKI) ${ }^{25,54}$ ) relevant to the above tests are also described in the Supplementary Note.

Unpredictable chronic mild stress and associated prophylactic and reversal antidepressant investigations. After a 2-week acclimation period, BALB/c male mice (8 weeks old) were individually housed and subjected to UCMS as described ${ }^{19}$. Stressors, typically wet bedding, tilted cages, lights on at night, crowding, difficult access to food, paired housing with an intruder, restraint or forced swim, were applied twice per day for a 2 -h period and overnight in a randomized order. No food deprivation was used. To avoid habituation to the stressor, the restraint and forced swimming stressors were introduced during the third week. Control (nonstressed) mice were group-housed in cages of five mice in a room distinct from that of the stressed mice. Throughout the UCMS protocol, the weights of the mice were measured every $5 \mathrm{~d}$. At the end of the chronic stress protocol, the emotional states of the mice were evaluated using the TST and the sucrose 
preference test as described ${ }^{19}$. The stress procedure was maintained with items compatible with behavioral testing.

Two variations of the protocol were used to assess pharmacological effects. They differ in respect to their total duration and the starting point of the injections of the compounds tested.

(i) Prophylactic protocol (21 d of UCMS; treatment throughout the 21-d period): In this protocol, we assessed the capacity of TDE to prevent stress-induced changes. For this purpose, TDE was administered prophylactically, starting from the first day of the UCMS stress (at a dose of $1 \mathrm{mg} / \mathrm{kg}$, i.p., daily) throughout the experiment. This protocol has been successfully used to show the prophylactic effects ${ }^{19}$ of reference antidepressants (fluoxetine) and of experimental compounds. Control (nonstressed) mice received saline or TDE ( $1 \mathrm{mg} / \mathrm{kg}$, i.p., daily) for $21 \mathrm{~d}$.

(ii) Reversal protocol (45 d of UCMS; treatment during the last 3 weeks): Antidepressant reversal protocols described previously in the literature ${ }^{55}$ were used with slight adaptations. Mice were subjected to the UCMS protocol for $45 \mathrm{~d}$ starting at Day 0. During the first 3 weeks, there was no treatment for any of the groups. From week 4 until the end of the protocol, mice were treated daily with saline or the compound being investigated, namely fluoxetine $(15 \mathrm{mg} / \mathrm{kg}$, i.p., daily) or TDE ( $1 \mathrm{mg} / \mathrm{kg}$, i.p., daily). Control (nonstressed) mice were left undisturbed for the first 3 weeks and received treatment the last $21 \mathrm{~d}$.

Definition of responder and nonresponder mice in the UCMS protocol of fluoxetine reversal. In the literature, it has been reported that fluoxetine administered in a reversal mode exerts a bimodal effect; it elicits a response in a subgroup of responder mice and has no effect on a distinct subgroup of nonresponders ${ }^{55}$. We therefore sought to distinguish between fluoxetine responders and nonresponders for subsequent qPCR determinations of Elk1 mRNA expression.

For this, at the end of the treatment we calculated a composite 'depression index' for UCMS-subjected, fluoxetine-treated mice. This index was based on responses in the TST and the sucrose preference test. $Z$-normalization and depression index computation was as described above (behavioral testing of AAVoverexpressing mice), except that UCMS-subjected, vehicle-treated mice were used as the control group for $Z$-score computation ${ }^{36}$.

UCMS-subjected, fluoxetine-treated mice were subsequently subdivided into two groups, responders versus nonresponders. The cutoff value was the average of the depression index for the UCMS-subjected, vehicle-treated mice.

Social defeat stress. Experimental mice were subjected to social defeat stress ${ }^{21}$ for 10 consecutive days. Every day, each experimental mouse was introduced into the home cage of an unfamiliar aggressive resident and was physically defeated. Resident mice were CD1 breeders selected for attack latencies reliably shorter than $30 \mathrm{~s}$ upon three consecutive screening tests. After $10 \mathrm{~min}$, residents and intruders were separated, but they were maintained in sensory contact for $24 \mathrm{~h}$ using a perforated plexiglass partition dividing the resident home cage in two halves. Every day, experimental mice were exposed to a new aggressor. Controls were similarly pair-housed, one on each side of a perforated plexiglass partition, and were handled daily.

In this protocol, we assessed the capacity of TDE to reverse changes induced by social defeat stress. After the end of the social defeat stress, the mice were assessed in the social approach-avoidance test. Then, they were separated in two treatment groups: mice that were treated with vehicle (saline) for $14 \mathrm{~d}$ and mice that were treated with TDE ( $1 \mathrm{mg} / \mathrm{kg}$, i.p., daily) for $14 \mathrm{~d}$. Social avoidance and anxiety (elevated plus maze) were assessed at the end of the 14-d treatment. Thus in this protocol, TDE was administered therapeutically (i.e., after social defeat and baseline behavioral characterization) in socially defeated mice. Experiments assessing the effects of TDE on the SD paradigm were replicated with at least three different cohorts of mice.

Social approach-avoidance test. Each mouse was introduced into an open field arena $(42 \times 42 \mathrm{~cm})$, and trajectory and behavior of the mouse was tracked for two consecutive sessions of $2.5 \mathrm{~min}$. During the first session ('no target mouse'), the open field contained an empty perforated plexiglass cage $(10 \times 6.5 \mathrm{~cm})$ located at one end. During the second session ('target'), the conditions were identical, except that a social target animal (an unfamiliar nonaggressive CD1 mouse) was introduced into the cage. For both sessions, the time spent in the interaction zone surrounding the perforated plexiglass enclosure was measured. Social approachavoidance is expressed as an interaction ratio: the time spent in the interaction zone during Session 2 over the time spent in the interaction zone during Session 1.

RNA and protein determinations in mice. Methods for qRT-PCR, radioactive in situ hybridization and FISH were as previously described ${ }^{56-58}$ Immunohistochemistry determinations were run with commercial antibodies described in the Nature Research Reporting Summary according to standard procedures. Details are given in the Supplementary Note.

In vivo electrophysiological recordings. Male $B A L B / c$ mice were anaesthetized with a mixture of air and isoflurane (CSP, Cournon d'Auvergne, France) during surgery and throughout the electrophysiological recordings using an anesthesia gas workstation (U410, Univentor Limited, Zejtun, Malta). Mice were held in a stereotactic frame (David Kopf Instruments, Tujunga, CA, USA) and maintained at a constant body temperature of $37.0 \pm 0.5^{\circ} \mathrm{C}$ (Harvard Apparatus, Les Ulis, France). A concentric bipolar stimulating electrode, made from $150-\mu$ m diameter stainless steel wire inserted into a 300- $\mu \mathrm{m}$ diameter microtube, was positioned in the medial perforant path (MPP, coordinates: $3 \mathrm{~mm}$ lateral to lambda, depth $\sim 1.5 \mathrm{~mm}$ from brain surface), and a glass micropipette recording electrode containing a silver wire immersed in saline was lowered into the dendritic layer of the ipsilateral DG or in the hilus ( $2 \mathrm{~mm}$ posterior to bregma, $1.6 \mathrm{~mm}$ lateral, $\sim 1.5 \mathrm{~mm}$ from brain surface). Electrode depth was adjusted to maximize the amplitude of evoked field potential. After surgery, low-frequency baseline stimuli (monophasic pulse, $60-\mu$ s pulse-width, $0.033 \mathrm{~Hz}$, delivered via a photically isolated constant current unit) were delivered to evoke a fEPSP. Responses were stored for off-line analyses of the maximum slope of the rising phase of the fEPSP and amplitude of the population spike as described previously ${ }^{59}$. After a stable response was established, LTP experiments were conducted, and negative-going fEPSPs were recorded from the dendritic layer of the DG. MPP test stimuli $(60 \mu \mathrm{s})$ were first delivered at $0.033 \mathrm{~Hz}$ for $30 \mathrm{~min}$ at an intensity to evoke a fEPSP slope at $50 \%$ of maximum to establish a baseline. A tetanus was then delivered to the MPP, which consisted of six series of six trains of six stimuli at $400 \mathrm{~Hz}$ with $200 \mathrm{~ms}$ between trains and $20 \mathrm{~s}$ between series. During the tetanus, pulse-width was doubled. This protocol has been shown to produce robust LTP in mice in in vivo experiments ${ }^{60}$. After the tetanus, low-frequency stimulation was resumed for $75 \mathrm{~min}$. Data are expressed as percent change in fEPSP slope relative to the pretetanus baseline.

Statistics. Statistical analyses were performed using the STATISTICA software. The results are expressed as mean \pm s.e.m. For the comparison of two independent groups, the Student's $t$-test was used. For comparisons involving more than two groups, multiple group comparisons were performed using one-way ANOVA, two-way factorial ANOVA, repeated-measures one-way ANOVA or repeatedmeasures two-way ANOVA depending on the experimental design. When ANOVA effects were significant, multiple group comparisons were followed by Duncan's or Fisher's LSD post hoc tests.

In all above analyses, we tested for homogeneity of variances using the Levene's test. When variances were not homogenous (due to the nature of the experiment and the variables) a nonparametric test (Mann-Whitney) was used for further analysis (for example, in the latent learning test in which a cut-off observation time is imposed for all mice, treatment-naive mice cluster at this cut-off, which is expected). For each experiment, the tests used, as well as the main effect and post hoc statistical significances, are as given in the appropriate figure legend.

Reporting Summary. Further information on experimental design is available in the Nature Research Reporting Summary linked to this article.

Data availability. The datasets that support the findings of this study are available from the corresponding author upon reasonable request.

\section{References}

41. Nolte, J. The Human Brain: An Introduction to its Functional Anatomy (Mosby/Elsevier, Philadelphia, 2009).

42. Uher, R. et al. An inflammatory biomarker as a differential predictor of outcome of depression treatment with escitalopram and nortriptyline. Am. J. Psychiatry 171, 1278-1286 (2014).

43. Bell, J. A., Kivimäki, M., Bullmore, E. T., Steptoe, A. \& Carvalho, L. A. Repeated exposure to systemic inflammation and risk of new depressive symptoms among older adults. Transl. Psychiatry 7, e1208 (2017).

44. Consoloni, J. L. et al. Serotonin transporter gene expression predicts the worsening of suicidal ideation and suicide attempts along a long-term follow-up of a major depressive episode. Eur. Neuropsychopharmacol. 28, 401-414 (2018).

45. Hervé, M. et al. Translational identification of transcriptional signatures of major depression and antidepressant response. Front. Mol. Neurosci. 10, 248 (2017).

46. Tzavara, E. T. et al. M4 muscarinic receptors regulate the dynamics of cholinergic and dopaminergic neurotransmission: relevance to the pathophysiology and treatment of related CNS pathologies. FASEB J. 18, 1410-1412 (2004).

47. Paxinos, G. \& Franklin, K. B. J. The Mouse Brain In Stereotaxic Coordinates (Gulf Professional Publishing, Houston, 2003).

48. Li, X., Need, A. B., Baez, M. \& Witkin, J. M. Metabotropic glutamate 5 receptor antagonism is associated with antidepressant-like effects in mice. J. Pharmacol. Exp. Ther. 319, 254-259 (2006).

49. Mombereau, C. et al. Genetic and pharmacological evidence of a role for $\mathrm{GABA}(\mathrm{B})$ receptors in the modulation of anxiety- and antidepressant-like behavior. Neuropsychopharmacology 29, 1050-1062 (2004).

50. Dulawa, S. C. \& Hen, R. Recent advances in animal models of chronic antidepressant effects: the novelty-induced hypophagia test. Neurosci. Biobehav. Rev. 29, 771-783 (2005).

51. Gur, T. L. et al. cAMP response element-binding protein deficiency allows for increased neurogenesis and a rapid onset of antidepressant response. J. Neurosci. 27, 7860-7868 (2007). 
52. Kruk-Slomka, M., Michalak, A., Budzynska, B. \& Biala, G. A comparison of mecamylamine and bupropion effects on memory-related responses induced by nicotine and scopolamine in the novel object recognition test in mice. Pharmacol. Rep. 66, 638-646 (2014).

53. Tzavara, E. T. et al. Endocannabinoids activate transient receptor potential vanilloid 1 receptors to reduce hyperdopaminergia-related hyperactivity: therapeutic implications. Biol. Psychiatry 59, 508-515 (2006).

54. Hancock, C. N. et al. Identification of novel extracellular signalregulated kinase docking domain inhibitors. J. Med. Chem. 48, 4586-4595 (2005).

55. Dournes, C., Beeské, S., Belzung, C. \& Griebel, G. Deep brain stimulation in treatment-resistant depression in mice: comparison with the CRF1 antagonist, SSR125543. Prog. Neuropsychopharmacol. Biol. Psychiatry 40, 213-220 (2013)
56. Moutsimilli, L. et al. Antipsychotics increase vesicular glutamate transporter 2 (VGLUT2) expression in thalamolimbic pathways. Neuropharmacology 54, 497-508 (2008)

57. Meffre, D. et al. Liver X receptors alpha and beta promote myelination and remyelination in the cerebellum. Proc. Natl. Acad. Sci. USA 112, 7587-7592 (2015)

58. Viereckel, T. et al. Midbrain gene screening identifies a new mesoaccumbal glutamatergic pathway and a marker for dopamine cells neuroprotected in Parkinson's disease. Sci. Rep. 6, 35203 (2016).

59. Morice, E. et al. Defective synaptic transmission and structure in the dentate gyrus and selective fear memory impairment in the Rsk 2 mutant mouse model of Coffin-Lowry syndrome. Neurobiol. Dis. 58, 156-168 (2013).

60. Errington, M. L., Bliss, T. V., Morris, R. J., Laroche, S. \& Davis, S. Long-term potentiation in awake mutant mice. Nature 387, 666-667 (1997). 


\section{natureresearch}

\section{Life Sciences Reporting Summary}

Nature Research wishes to improve the reproducibility of the work that we publish. This form is intended for publication with all accepted life science papers and provides structure for consistency and transparency in reporting. Every life science submission will use this form; some list items might not apply to an individual manuscript, but all fields must be completed for clarity.

For further information on the points included in this form, see Reporting Life Sciences Research. For further information on Nature Research policies, including our data availability policy, see Authors \& Referees and the Editorial Policy Checklist.

Please do not complete any field with "not applicable" or n/a. Refer to the help text for what text to use if an item is not relevant to your study. For final submission: please carefully check your responses for accuracy; you will not be able to make changes later.

\section{- Experimental design}

1. Sample size

Describe how sample size was determined.

\section{Data exclusions}

Describe any data exclusions.
There was no prior statistical determination of sample size but sample sizes are consistent to those reported in the literature in similar studies. Sample sizes are reported in Figure legends or Figures

In correlation studies outliers with values of more/less than 2SD were excluded. This criterion was pre-established.

For the replicatory cohort: Based on the involvement of our protein in immunological process and the potentially confounding interaction between chronic mild inflammation and antidepressant response (Uher et al., 2014), we excluded form this analysis all patients with serum C-reactive -protein (CRP) $>5 \mathrm{mg} / \mathrm{L}$ at inclusion and/or patients with a variation in CRP level exceeding three-fold (a criterion for inflammation (Bell et al;, 2017) between inclusion and week-8.

Animal studies: Reasons for exclusion were the following: (i) In chronic animal study protocols (UCMS, NIH, SD) mice were excluded from the statistical analysis if they died, got injured from injection in pharmacological studies, or became seriously ill during the protocol; (ii) in all behavioral experiments mice were excluded from the analysis if their behavior was incompatible with the aim of the test (i.e. mice climbing over their tail in the TST; mice that did not drink at all during the sucrose preference test). These two criteria were preestablished based on previous experience and experiments. For both criteria the occurrences were rare and evenly distributed across groups.

\section{Replication}

Describe the measures taken to verify the reproducibility of the experimental findings.

\section{Randomization}

Describe how samples/organisms/participants were allocated into experimental groups.

\section{Blinding}

Describe whether the investigators were blinded to group allocation during data collection and/or analysis.

There was no blinding for animal studies, because of the nature of the experiments. In most cases RNA and protein determinations were performed by experimenters that were unaware of the experimental group of the animals.

No randomization table was used in animal studies. All animals were male adults (3-6 months of age) that were commercially purchased and assigned pseudorandomly to experimental groups upon arrival.

Note: all in vivo studies must report how sample size was determined and whether blinding and randomization were used. 


\section{Statistical parameters}

For all figures and tables that use statistical methods, confirm that the following items are present in relevant figure legends (or in the Methods section if additional space is needed).

$\mathrm{n} / \mathrm{a} \mid$ Confirmed

\ The exact sample size $(n)$ for each experimental group/condition, given as a discrete number and unit of measurement (animals, litters, cultures, etc.)

$\triangle$ A description of how samples were collected, noting whether measurements were taken from distinct samples or whether the same

sample was measured repeatedly

$\searrow$ A statement indicating how many times each experiment was replicated

The statistical test(s) used and whether they are one- or two-sided

$\triangle$ Only common tests should be described solely by name; describe more complex techniques in the Methods section.

$\bigotimes$ A description of any assumptions or corrections, such as an adjustment for multiple comparisons

Test values indicating whether an effect is present

$\triangle$ Provide confidence intervals or give results of significance tests (e.g. P values) as exact values whenever appropriate and with effect sizes noted.

$\bigotimes$ A clear description of statistics including central tendency (e.g. median, mean) and variation (e.g. standard deviation, interquartile range)

$\searrow$ Clearly defined error bars in all relevant figure captions (with explicit mention of central tendency and variation)

See the web collection on statistics for biologists for further resources and guidance.

\section{- Software}

Policy information about availability of computer code

\section{Software}

Describe the software used to analyze the data in this study.

SPSS Software; Statistica Software; Sequence Detection System (SDS) software; the RQ Manager software (Applied Biosystems); MCID Analysis Software; NDP view2 software;

For manuscripts utilizing custom algorithms or software that are central to the paper but not yet described in the published literature, software must be made available to editors and reviewers upon request. We strongly encourage code deposition in a community repository (e.g. GitHub). Nature Methods guidance for providing algorithms and software for publication provides further information on this topic.

\section{- Materials and reagents}

Policy information about availability of materials

\section{Materials availability}

Indicate whether there are restrictions on availability of unique materials or if these materials are only available for distribution by a third party.
The Peptide TAD DEF Elk (TDE) used in the present article and its application in depression are protected by the published patents WO 2006/087242 and WO2010/037841. This does not alter our adherence to Nature Publishing Group policies on sharing data and materials. 


\section{Antibodies}

Describe the antibodies used and how they were validated for use in the system under study (i.e. assay and species).
Primary antibodies

1. Phospho-p44/42 MAP kinase (1:500; Cell Signaling \#9101) antibody detects endogenous levels of p44 and p42 MAP Kinase (Erk1 and Erk2) when phosphorylated either individually or dually at Thr202 and Tyr204 of Erk1 (Thr185 and Tyr187 of Erk2). The antibody does not cross-react with the corresponding phosphorylated residues of either JNK/SAPK or p38 MAP Kinase, and does not cross-react with non-phosphorylated Erk1/2.

https://www.cellsignal.com/products/primary-antibodies/phospho-p44-42-mapk-erk1-2thr202-tyr204-antibody/9101

2. Phospho-MSK1 (1:500; Cell Signaling \#9595) antibody detects endogenous levels of MSK1 only when phosphorylated at Thr581. This antibody does not cross-react with MSK1 phosphorylated at other sites, nor does it detect phosphorylated RSK1, RSK2 or RSK3. https://www.cellsignal.com/products/primary-antibodies/phospho-msk1-thr581antibody/9595

3. p-Elk-1 (B-4; 1:200; Santa Cruz sc-8406) is a mouse monoclonal antibody raised against a sequence containing Ser 383 phosphorylated Elk-1 of human origin.

https://www.scbt.com/scbt/product/p-elk-1-antibody-b-4

4. Egr-1 (588; 1:200; Santa Cruz sc-110) is a rabbit polyclonal IgG, epitope mapping at the Cterminus of Egr-1 of human origin

5. https://www.scbt.com/scbt/product/egr-1-antibody-588

6. c-Fos (4; 1:200; Santa Cruz sc-52) is a rabbit polyclonal IgG, epitope mapping at the Nterminus of c-Fos of human origin

https://www.scbt.com/scbt/product/c-fos-antibody-4

7. Elk-1 (I-20; 1:50; Santa Cruz sc-355) is a rabbit polyclonal IgG, epitope mapping at the Cterminus of Elk-1 of human origin

https://www.scbt.com/scbt/product/elk-1-antibody-i-20

Secondary antibodies

1. Cy3- conjugated anti-rabbit (1:500; GE Healthcare 29038276)

https://www.gelifesciences.com/shop/amersham-wb-cy3-cy5-secondary-antibodiesp-01523?current $=29038276$

2. Cy3- conjugated anti-mouse (1:400; Jackson ImmunoResearch 115-165-100) https://www.jacksonimmuno.com/catalog/products/115-165-146

3. biotinylated goat anti-rabbit antibody (1:800; Vector Laboratories BA-1000) https://vectorlabs.com/biotinylated-goat-anti-rabbit-igg-antibody.html 4. A+B reagent (Vector Laboratories PK-4000). https://vectorlabs.com/vectastain-abc-kit-standard.html

No eukaryotic cell lines used

No eukaryotic cell lines used

No eukaryotic cell lines used

No eukaryotic cell lines used

d. If any of the cell lines used are listed in the database of commonly misidentified cell lines maintained by ICLAC, provide a scientific rationale for their use.

\section{- Animals and human research participants}

Policy information about studies involving animals; when reporting animal research, follow the ARRIVE guidelines

\section{Description of research animals}

Provide all relevant details on animals and/or animal-derived materials used in the study.
All mice used were male adults (which is 3-6 months old). The following strains were used: C57BI6, CD1 and BALB/CJico. All mice were obtained from commercial vendors

Policy information about studies involving human research participants

\section{Description of human research participants}

Describe the covariate-relevant population characteristics of the human research participants. a) Post mortem studies: all samples were from male subjects. Samples were matched between groups for age, tissue pH, postmortem interval (PMI) and RNA integrity number (RIN)

b) Clinical cohorts: i) Discovery cohort: 14 healthy controls ( $42.7 \%$ female, mean age $=38.1 \pm 9.4 \mathrm{SD})$ and 28 patients with MDD (67.9\% female, mean age $=45.0 \pm$ 10.9 SD) have been included in the analysis; ii) Replication cohort: 26 patients with MDE (16 women, $61.5 \%)$ with a mean age of 41.7 ( $\pm 17.0 \mathrm{SD}$ ) and a mean depressive symptoms severity of $23( \pm 3.2 \mathrm{SD}$ ) have been included in the analysis. 